\title{
Efecto de la aplicación foliar suplementaria de calcio sobre la producción de melón (Cucumis melo L.)
}

\section{Effect of the supplementary foliar application of calcium on melon (Cucumis melo L.) production}

José Eladio Monge-Pérez ${ }^{1}$, Michelle Loría-Coto²

Fecha de recepción: 18 de agosto de 2019

Fecha de aprobación: 2 de diciembre de 2019

Monge-Pérez, J; Loría-Coto, M. Efecto de la aplicación foliar suplementaria de calcio sobre la producción de melón ( $\mathrm{Cu}$ cumis melo L.). Tecnología en Marcha. Vol. 33-4. Octubre-

Diciembre 2020. Pág 89-102.

doi) https://doi.org/10.18845/tm.v33i4.4556

1 Ingeniero agrónomo. Docente de Sede de Guanacaste, e Investigador de Estación Experimental Agrícola Fabio Baudrit Moreno, Universidad de Costa Rica, Costa Rica. Correo electrónico: melonescr@yahoo.com.mx.

2 Economista agrícola. Docente e Investigadora de la Escuela de Ciencias Exactas y Naturales, Universidad Estatal a Distancia, Costa Rica. Correo electrónico: michelle_loria@yahoo.com. 


\title{
Palabras clave
}

Cucumis melo; melón; peso del fruto; 'Brix, firmeza de la pulpa del fruto; rendimiento; calidad; Pseudoperonospora cubensis.

\section{Resumen}

Se evaluó el efecto de la aplicación foliar suplementaria de calcio, sobre la producción de melón Amarillo cv. Natal F-1, en Lepanto, Puntarenas. Los tratamientos evaluados fueron: JMX-Ca (4,22 kg CaO/ha), JMX-Ca (2,41 kg CaO/ha), Manvert-Ca (4,22 kg CaO/ha), y un testigo (agua). La cosecha inició a los 58 días después del trasplante (ddt) para todos los tratamientos. Los datos muestran una importante variabilidad entre los tratamientos en cuanto al número de frutos exportables por metro lineal $(1,79-2,06)$, rendimiento exportable $(13638-16126 \mathrm{~kg} /$ ha), producción exportable (1318 - 1556 cajas/ha), peso promedio del fruto (1364,3 - 1433,0 g), porcentaje de sólidos solubles totales (10,90 - 11,88 ${ }^{\circ}$ Brix), firmeza de la pulpa del fruto (26,94 - 28,38 N), severidad máxima de mildiú velloso (Pseudoperonospora cubensis) a los 50 ddt (3,83 - 4,17; escala 1-5), e incidencia de colapso de la planta a los 58 ddt (0 - 1,85 $\%)$. Solamente se presentaron diferencias significativas entre tratamientos para el porcentaje de sólidos solubles totales; el tratamiento de JMX-Ca a 4,22 kg CaO/ha obtuvo un mayor valor para esta característica (11,88 ${ }^{\circ}$ Brix) en comparación a JMX-Ca a 2,41 kg CaO/ha y a Manvert$\mathrm{Ca}$ a $4,22 \mathrm{~kg} \mathrm{CaO} / \mathrm{ha}$ (entre 10,90 y 11,15 ${ }^{\circ}$ Brix), pero no fue diferente del testigo (11,45 'Brix). Se concluye que, bajo las condiciones en que se desarrolló el ensayo, no se justifica económicamente la aplicación foliar suplementaria de calcio.

\section{Keywords}

Cucumis melo; melon; fruit weight; 'Brix; fruit flesh firmness; yield; quality; Pseudoperonospora cubensis.

\begin{abstract}
The effect of supplementary foliar application of calcium on production of Amarillo type melon cv. Natal F-1, was evaluated in Lepanto, Puntarenas. Evaluated treatments were: JMX-Ca $(4,22$ $\mathrm{kg} \mathrm{CaO} / \mathrm{ha})$, JMX-Ca (2,41 kg CaO/ha), Manvert-Ca (4,22 kg CaO/ha), and control (water). The harvest started 58 days after transplant (dat) for all treatments. Data show an important variability among treatments with respect to number of exportable fruits per linear meter $(1,79$ 2,06), exportable yield (13638 - $16126 \mathrm{~kg} / \mathrm{ha})$, exportable production (1318 - 1556 boxes/ha), mean fruit weight $(1364,3-1433,0 \mathrm{~g})$, percentage of total soluble solids $\left(10,90-11,88{ }^{\circ} \mathrm{Brix}\right)$, fruit flesh firmness $(26,94-28,38 \mathrm{~N})$, maximum downy mildew (Pseudoperonospora cubensis) severity at 50 dat $(3,83-4,17 ; 1-5$ scale), and plant collapse incidence at 58 dat $(0-1,85$ $\%)$. Differences between treatments were significant only for the percentage of total soluble solids; JMX-Ca at 4,22 $\mathrm{kg} \mathrm{CaO} / \mathrm{ha}$ rendered the highest value for this characteristic $\left(11,88^{\circ} \mathrm{Brix}\right)$ compared to JMX-Ca at 2,41 kg CaO/ha and Manvert-Ca at 4,22 kg CaO/ha (10,90 and 11,15 $\left.{ }^{\circ} \mathrm{Brix}\right)$. But the difference was not significant compared to the control treatment $\left(11,45^{\circ} \mathrm{Brix}\right)$. In conclusion, there is no economical justification for the supplementary foliar application of calcium under the growing conditions of the trial.
\end{abstract}




\section{Introducción}

En 2011, el melón (Cucumis melo L.) fue el quinto producto agrícola de exportación de Costa Rica (2,8 \% del total), luego de banano, piña, café oro y plantas ornamentales, y generó divisas por un total de 66,9 millones de dólares. Los principales tipos de melón producidos en Costa Rica para la exportación son: Harper, Cantaloupe, Amarillo, Honey Dew, Galia, Piel de Sapo, Charentais, y Orange Flesh [1]. En el caso del melón Amarillo, en 2012 se cultivaban 2109 ha, lo que representó un 46 \% del área cultivada de melón en el país; el principal mercado para este tipo de melón es Europa [2].

El melón es un cultivo de ciclo corto, que requiere una aplicación intensiva de insumos para el logro de rendimientos altos. Además, por tratarse principalmente de un cultivo de exportación en Costa Rica, es muy importante lograr la producción de fruta de alta calidad, que satisfaga los estándares de los mercados extranjeros.

El melón demanda una cantidad importante de nutrientes; se ha estimado que una hectárea de este cultivo consume en total $114 \mathrm{~kg}$ de calcio (Ca), $97 \mathrm{~kg}$ de potasio (K), $83 \mathrm{~kg}$ de nitrógeno (N), $24 \mathrm{~kg}$ de magnesio (Mg), y $15 \mathrm{~kg}$ de fósforo (P). En el caso del calcio, la cantidad consumida de dicho elemento por este cultivo es muy alta, así como la desproporción entre la cantidad que requiere la parte vegetativa (90 \%) y la que consume el fruto (10 \%). Sin embargo, como el calcio es un elemento que no se transloca en modo alguno, los aproximadamente $10 \mathrm{~kg} / \mathrm{ha}$ que requieren los frutos deben ingresar en los últimos 20 días de llenado de fruto, y además, es muy importante que lo hagan [3]. Para lograr este cometido, una de las principales herramientas que tiene a disposición el agricultor es la aplicación foliar de este elemento, especialmente en forma de quelatos [4].

El objetivo de este trabajo fue evaluar el efecto de la aplicación foliar suplementaria de calcio, sobre el rendimiento y la calidad en el cultivo de melón.

\section{Materiales y métodos}

El ensayo se realizó en una finca ubicada en Lepanto, Puntarenas, administrada por la empresa M y M Internacional S. A., la cual se dedicaba a la producción y exportación de melón y sandía a Europa. El ensayo se ubicó en el lote 6-1 de dicha finca; el trasplante se realizó el día 27 de diciembre de 2015, con plántulas del híbrido de melón amarillo (Cucumis melo L.), cv. Natal F-1.

El cultivo se sembró en camas de 1,0 m de ancho, cubiertas con plástico. La distancia entre hileras fue de 1,8 m, y la distancia entre plantas fue de 0,3 m, por lo que la densidad de siembra correspondió a 18519 plantas por hectárea.

La fertilización implementada normalmente por la finca consiste en la aplicación de fertilizante granulado al momento de la siembra, junto con el aporte de fertilizantes solubles vía fertirriego a lo largo del ciclo del cultivo. Además, en forma complementaria, se realizan aplicaciones foliares de fertilizantes. La fuente de calcio empleada a nivel foliar por la finca corresponde al fertilizante líquido Amino Grow Calcio, que contiene 7 \% p/v de CaO; 4,3 \% p/v de N; 1,41 \% $\mathrm{p} / \mathrm{v}$ de aminoácidos, así como reguladores de crecimiento (auxinas, giberelinas y citoquininas); se hicieron 5 aplicaciones de este producto, a lo largo del ciclo del cultivo.

En forma suplementaria (adicional a los fertilizantes aplicados normalmente por la finca), para el presente ensayo se aplicaron los siguientes fertilizantes foliares:

1) JMX-Ca: fertilizante foliar sólido, en presentación de polvo muy fino, color blanco, y cuya composición es: 40,2% p/p de CaO; 2440 ppm de MgO; 190 ppm de Fe; y 240 ppm de Si. 
2) Manvert-Ca: fertilizante foliar líquido, de color café oscuro, y cuya composición es: 20,2\% $\mathrm{p} / \mathrm{v}$ de $\mathrm{CaO}$; el agente acomplejante es ácido lignosulfónico.

Los tratamientos, aplicados en forma suplementaria al programa de fertilización normal de la finca, se muestran en el cuadro 1.

Cuadro 1. Tratamientos aplicados en el ensayo.

\begin{tabular}{|c|c|c|c|c|}
\hline \multicolumn{2}{|c|}{ Tratamiento } & \multirow{2}{*}{$\begin{array}{c}\text { Fechas de aplicación } \\
(\mathrm{ddt})\end{array}$} \\
\hline \multirow{2}{*}{ Número } & Fertilizante & Dosis de CaO (kg/ha) & Dosis del fertilizante & $12 ; 26 ; 40 ; 50$ \\
\hline 1 & Testigo (agua) & 0 & 0 & $12 ; 26 ; 40 ; 50$ \\
\hline 3 & JMX-Ca & 4,22 & $10,5 \mathrm{~kg} / \mathrm{ha}$ & $26 ; 40$ \\
\hline 4 & JMX-Ca & 2,41 & $20,8 \mathrm{~kg} / \mathrm{ha}$ & $12 ; 26 ; 40 ; 50$ \\
\hline
\end{tabular}

Nota: * = días después de trasplante.

Todos los tratamientos se hicieron mediante aspersión foliar. El tratamiento 1 (testigo) consistió en la aplicación de agua limpia. En el tratamiento 2 se realizaron cuatro aplicaciones del fertilizante JMX-Ca; la dosis de la primera aplicación fue de 1,5 kg/ha, y la dosis de las otras tres aplicaciones fue de $3,0 \mathrm{~kg} / \mathrm{ha}$ cada una. En el tratamiento 3 se hicieron dos aplicaciones de JMX-Ca, a una dosis de 3,0 kg/ha cada una. En el tratamiento 4 se realizaron cuatro aplicaciones del fertilizante Manvert-Ca; la dosis de la primera aplicación fue de 2,98 I/ha, y la dosis de las otras tres aplicaciones fue de 5,97 I/ha cada una. De este modo, tanto el tratamiento 2 como el tratamiento 4 aportaron en total 4,22 kg/ha de CaO, y el tratamiento 3 aportó 2,41 kg/ha de CaO.

Los tratamientos se aplicaron en todos los casos con una bomba de espalda marca Carpi, modelo Spray Mec, de 18 litros de capacidad. Entre la aplicación de un tratamiento y el siguiente, se realizó un lavado adecuado de la bomba, con agua limpia. El volumen total de caldo aplicado al cultivo correspondió a 231 I/ha para las aplicaciones realizadas a los 12 y 26 ddt, y a 462 l/ha para las aplicaciones hechas a los 40 y 50 ddt, debido a la mayor cantidad de follaje presente en esos momentos en el cultivo.

Para la aplicación de todos los tratamientos, se añadió el coadyuvante Agrex RP 25,7 SL, a una dosis de 0,5 ml/litro. Este producto contiene 16,2 \% p/v de alquil-aril-éter-poliglicólico; 4,0\% p/v de dioctil sulfosuccinato; y $5,5 \% \mathrm{p} / \mathrm{v}$ de glicol.

Las variables evaluadas fueron:

a) Edad al inicio de cosecha (ddt): se calculó según los días transcurridos desde el trasplante hasta el inicio de la cosecha, para cada tratamiento.

b) Número de frutos por metro lineal: se contabilizó el número de frutos exportables por parcela útil, y se dividió entre $8 \mathrm{~m}$ (longitud de la cama de la parcela útil). Se consideraron como frutos no aptos para exportación aquellos con deformidades, decoloraciones, o daños por plagas, enfermedades o quema por sol.

c) Rendimiento exportable (kg/ha): se obtuvo a partir del peso total de los frutos exportables y de la densidad de siembra, y se calculó el rendimiento por hectárea. Se utilizó una balanza electrónica marca Ocony, modelo TH-I-EK, con una capacidad de 5000,0 × 0,1 g. 
d) Producción exportable (cajas/ha): se obtuvo al relacionar el número de frutos según su tamaño y peso, y a partir de este dato y de la densidad de siembra, se calculó el número total de cajas exportables de $10 \mathrm{~kg}$ producidas en una hectárea.

e) Peso promedio del fruto ( $g$ ): se obtuvo al dividir el peso total de los frutos exportables por parcela, entre el número total de los mismos.

f) Distribución porcentual de tamaño de los frutos: se obtuvo al estimar el porcentaje de frutos ubicados en cada categoría de tamaño, según su peso. El tamaño del fruto se expresa de acuerdo con el número de frutos que caben en una caja de $10 \mathrm{~kg}$. Así, entre mayor sea el número que identifica el tamaño del fruto, menor será el tamaño del mismo. Para hacer esta estimación se utilizó el rango de peso que se muestra en el cuadro 2.

Cuadro 2. Rango de peso del fruto, según la categoría de tamaño.

\begin{tabular}{|c|c|}
\hline Categoría de tamaño del fruto & Rango de peso del fruto $(\mathrm{g})$ \\
\hline 4 & $2223-2750$ \\
\hline 5 & $1819-2222$ \\
\hline 6 & $1539-1818$ \\
\hline 7 & $1334-1538$ \\
\hline 8 & $1177-1333$ \\
\hline 9 & $1053-1176$ \\
\hline 10 & $870-1052$ \\
\hline 12 & $800-869$ \\
\hline
\end{tabular}

g) Porcentaje de sólidos solubles totales ( ${ }^{\circ}$ Brix): se evaluó el porcentaje de sólidos solubles totales de la pulpa del fruto, en una muestra de cinco frutos exportables por parcela, y se obtuvo el promedio. Se utilizó un refractómetro manual marca Atago, modelo N-1a, con una escala de $0,0-32,0 \pm 0,2^{\circ}$ Brix.

h) Firmeza de la pulpa del fruto $(N)$ : se evaluó la firmeza de la pulpa del fruto, en una muestra de cinco frutos exportables por parcela, y se calculó el promedio. Se utilizó un penetrómetro marca Effegi, modelo FT-327, con una escala de 0,0 - 13,0 \pm 0,1 kg-fuerza, y los valores obtenidos se multiplicaron por un factor de conversión de 9,807 para calcular el dato en Newtons (N); se utilizó el puntero del penetrómetro cuya base mide 7,5 mm de ancho.

i) Severidad máxima de mildiú velloso: se realizaron cuatro evaluaciones fitosanitarias (a los 12 ddt, 26 ddt, 40 ddt y 50 ddt), a nivel cuantitativo en todas las plantas de cada parcela, para determinar la severidad máxima de la enfermedad mildiú velloso (Pseudoperonospora cubensis), según la escala que se muestra en el cuadro 3.

j) Incidencia de colapso de la planta: se realizaron cinco evaluaciones fitosanitarias (a los 12 ddt, 26 ddt, 40 ddt, 50 ddt y 58 ddt) a nivel cuantitativo, para determinar el porcentaje de plantas colapsadas por enfermedades causadas por hongos fitopatógenos en el cuello de la planta (tales como Fusarium spp., Sclerotium rolfsii, Mycosphaerella spp., entre otros). Se evaluaron todas las plantas de cada parcela. 
Cuadro 3. Escala de evaluación de la severidad de mildiú velloso en melón.

\begin{tabular}{|c|c|}
\hline Número de lesiones necróticas por hoja & Escala \\
\hline 0 & 1 \\
\hline $1-3$ & 2 \\
\hline $4-6$ & 3 \\
\hline $7-10$ & 4 \\
\hline Más de 10 & 5 \\
\hline
\end{tabular}

k) Relación beneficio:costo del tratamiento: se calculó a partir del costo adicional de los fertilizantes aplicados, el precio de venta de la caja de melón de exportación, y el aumento o reducción adicional en el rendimiento, en comparación con el testigo (el cual se tomó como el tratamiento base). El precio considerado para el JMX-Ca fue de 7238 colones/ kg, para el Manvert-Ca fue de 2399 colones/litro, y para la caja de melón de exportación fue de 4048 colones/caja.

Además, para cada tratamiento se realizó un análisis foliar orientativo; se tomó una muestra compuesta de 30 hojas maduras (quinta hoja a partir del brote de la rama) para cada tratamiento (cinco hojas por cada parcela útil), y se realizó un análisis químico foliar completo, en el Centro de Investigaciones Agronómicas de la Universidad de Costa Rica.

Se utilizó un diseño experimental de bloques completos al azar, con seis repeticiones y cuatro tratamientos. Cada bloque consistió en una hilera de plantas de 48 metros de longitud, ubicado en la zona central del lote 6-1 de la finca. Cada bloque se separó del otro por medio de una hilera de plantas, que sirvió de borde entre bloques. La parcela total consistió en una sección de 12 metros lineales de cama, y la parcela útil consistió en la sección central de dicha parcela, con una longitud de 8 metros lineales de cama.

Para las variables cuantitativas de rendimiento, calidad, y daño por enfermedades, se realizó un análisis de variancia para establecer diferencias significativas entre tratamientos, y se usó la prueba LSD Fisher $(\mathrm{p} \leq 0,05)$ para clasificar los resultados de los tratamientos.

\section{Resultados y discusión}

La cosecha inició el 23 de febrero de 2016 (58 ddt), y finalizó el 28 de febrero de 2016 (63 ddt), para todos los tratamientos. No se observaron diferencias en el inicio de la madurez (precocidad) de los frutos entre tratamientos.

En la figura 1 se presentan los datos para el número de frutos exportables por metro lineal, según los diversos tratamientos evaluados. El tratamiento 2 mostró el mayor valor para esta variable con 2,06 frutos $/ \mathrm{m}$, y el menor valor correspondió al tratamiento 3 (1,79 frutos $/ \mathrm{m})$. Sin embargo, las diferencias encontradas entre tratamientos no fueron estadísticamente significativas.

En dos ensayos realizados en Brasil con melón Amarillo, no hubo diferencias en el número de frutos por planta (y por lo tanto, en el número de frutos por metro lineal) entre el testigo y el tratamiento de aplicación foliar de calcio [5], [6]; en el presente ensayo se obtuvo un resultado similar.

En la figura 2 se muestran los resultados del rendimiento exportable de melón. 


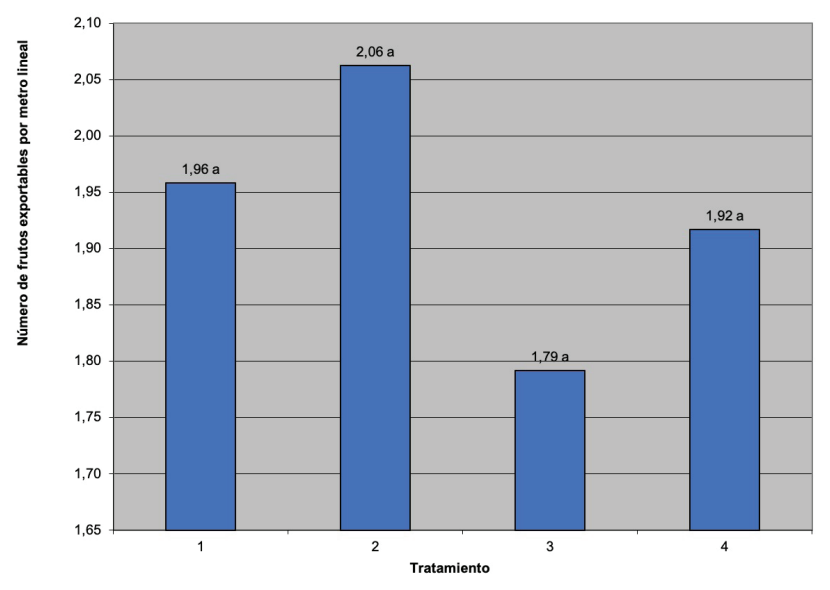

Figura 1. Número de frutos exportables por metro lineal, según tratamiento.

Nota: elaboración propia, a partir de los datos obtenidos. Medias seguidas de una misma letra no se consideran distintas según la prueba de LSD Fisher $(p \leq 0,05)$.

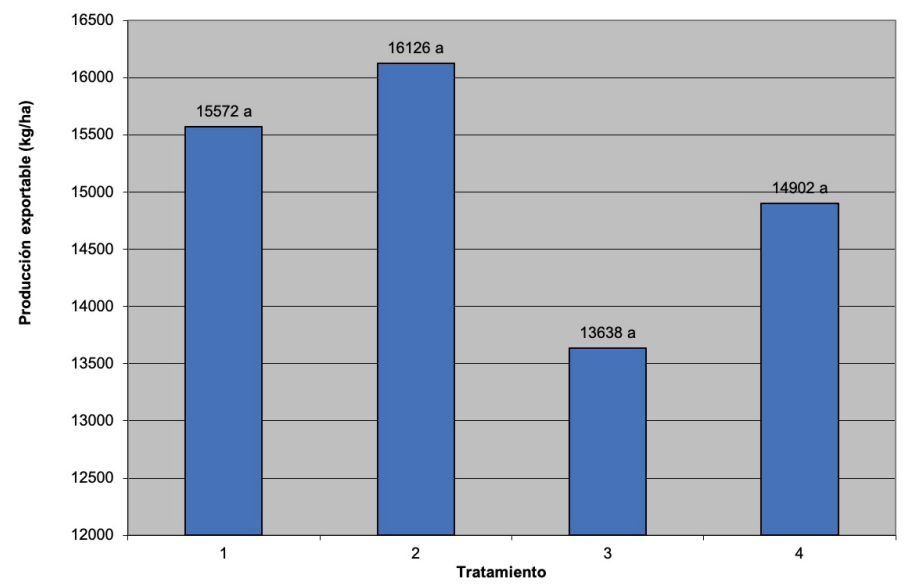

Figura 2. Rendimiento exportable de melón (kg/ha), según tratamiento.

Nota: Medias seguidas de una misma letra no se consideran distintas según la prueba de LSD Fisher $(p \leq 0,05)$.

El tratamiento 2 mostró el mayor rendimiento en este ensayo con $16126 \mathrm{~kg} / \mathrm{ha}$, y el menor rendimiento correspondió al tratamiento 3 (13638 kg/ha). Sin embargo, no se hallaron diferencias estadísticamente significativas entre tratamientos para esta variable. Los resultados obtenidos en el presente ensayo son inferiores a los rendimientos informados para exportación de melón en Costa Rica, de entre 17781 y 27516 kg/ha [2].

En un ensayo con melón Amarillo en Brasil, la aplicación foliar de calcio aumentó en forma significativa el rendimiento comercial para el híbrido Gold Mine en comparación al testigo, pero para el híbrido 10-00 no se presentaron diferencias con el testigo [5]; en el presente ensayo se obtuvo el mismo resultado que el hallado por dichos autores con el híbrido de melón 10-00.

En la figura 3 se presenta la producción exportable de melón, en cajas por hectárea. El tratamiento 2 presentó el mayor rendimiento con 1556 cajas/ha, y el tratamiento 3 mostró la menor producción (1318 cajas/ha). Pero las diferencias encontradas entre tratamientos no fueron estadísticamente significativas. 


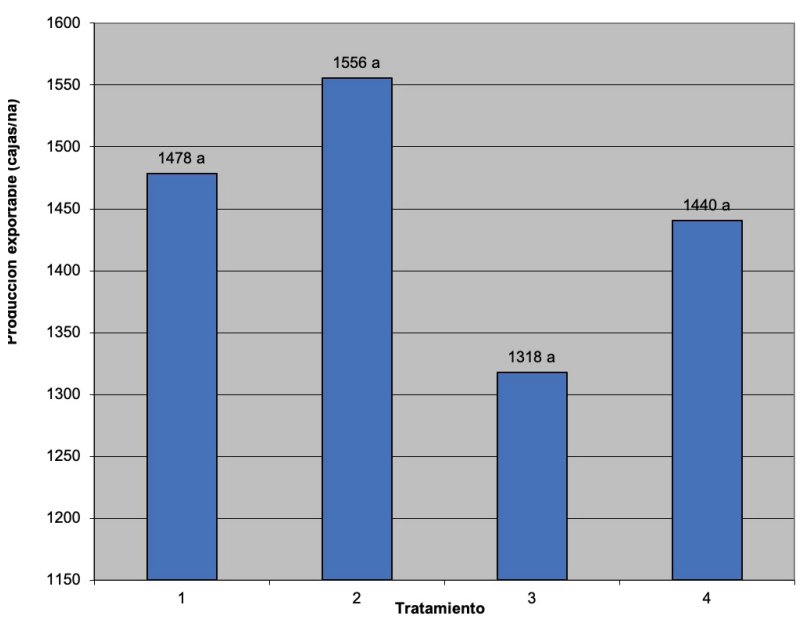

Figura 3. Producción exportable de melón (cajas/ha), según tratamiento.

Nota: Medias seguidas de una misma letra no se consideran distintas según la prueba de LSD Fisher $(p \leq 0,05)$.

Es conveniente resaltar que el rendimiento del cultivo en este ensayo fue bastante bajo, dado que los rendimientos normales para la zona y para esta variedad de melón, generalmente es cercano a las 2500 cajas/ha (J. E. Monge-Pérez, datos sin publicar).

En la figura 4 se muestran los datos del peso promedio del fruto, según el tratamiento evaluado. El tratamiento 2 produjo los frutos con mayor peso $(1433,0 \mathrm{~g})$, y el menor valor para esta variable se obtuvo con el tratamiento $3(1364,3 \mathrm{~g})$. Sin embargo, no se presentaron diferencias estadísticamente significativas entre tratamientos para esta variable.

En otro ensayo realizado en Argentina con melón Honey Dew, en cuatro localidades no se hallaron diferencias significativas en el peso del fruto entre el testigo y los tratamientos en que se aplicaron tres diferentes fuentes de calcio vía foliar; pero en otra localidad sí se hallaron diferencias para esta variable entre dos de las fuentes de calcio entre sí, pero no entre dichas fuentes y el testigo [7]; los resultados obtenidos en el presente ensayo son similares a los hallados por esos autores en las cuatro localidades supracitadas.

Sin embargo, en una investigación con melón Amarillo realizada en Brasil, el peso del fruto sí aumentó con la aplicación foliar de calcio, en comparación con el testigo [6].

En el cuadro 4 se presentan los datos de la distribución porcentual de tamaño de los frutos. El tratamiento 2 concentró la mayor proporción de frutos de tamaño 6, 7 y 8 juntos (68,69 \% de los frutos totales), que corresponden a los que tienen mayor demanda en el mercado en Europa, seguido por el tratamiento $3(61,63 \%)$, el tratamiento $4(58,70 \%)$, y por último el tratamiento $1(58,51 \%)$. Sin embargo, no se hallaron diferencias significativas entre tratamientos para la cantidad de frutos 6,7 y 8 juntos (no se muestran los datos del análisis estadístico). 


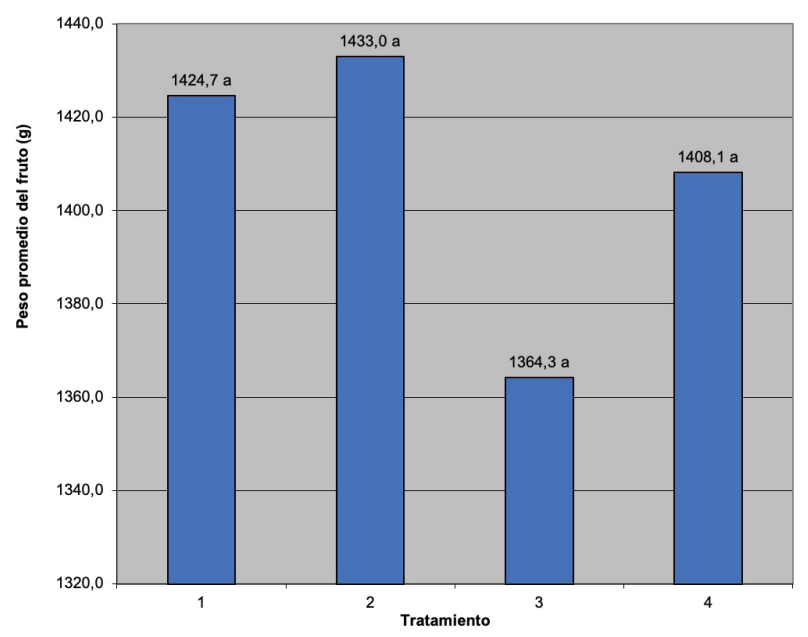

Figura 4. Peso promedio del fruto $(\mathrm{g})$, según tratamiento.

Nota: Medias seguidas de una misma letra no se consideran distintas según la prueba de LSD Fisher $(p \leq 0,05)$.

Cuadro 4. Distribución porcentual de tamaño de los frutos.

\begin{tabular}{|c|c|c|c|c|c|c|c|c|}
\hline \multirow{2}{*}{ Tratamiento } & \multicolumn{8}{|c|}{ Categoría de tamaño del fruto (valores en porcentaje) } \\
\cline { 2 - 9 } & 4 & 5 & 6 & 7 & 8 & 9 & 10 & 12 \\
\hline 1 & 3,19 & 9,57 & 24,47 & 22,34 & 11,70 & 13,83 & 10,64 & 4,26 \\
\hline 2 & 1,01 & 7,07 & 26,26 & 27,28 & 15,15 & 9,09 & 9,09 & 5,05 \\
\hline 3 & 0,00 & 9,30 & 19,77 & 24,42 & 17,44 & 9,30 & 19,77 & 0,00 \\
\hline 4 & 1,09 & 13,04 & 17,39 & 22,83 & 18,48 & 9,78 & 16,30 & 1,09 \\
\hline
\end{tabular}

En la figura 5 se muestran los resultados para el porcentaje de sólidos solubles totales ( ${ }^{\circ}$ Brix), según el tratamiento. Esta fue la única variable en que sí existieron diferencias estadísticamente significativas entre tratamientos; el mayor valor se presentó con el tratamiento 2 (11,88 $\left.{ }^{\circ} \mathrm{Brix}\right)$, y el menor valor lo obtuvo el tratamiento 3 (10,90 ${ }^{\circ}$ Brix). No se presentaron diferencias significativas entre el tratamiento 2 y el tratamiento 1 (testigo, 11,45 ${ }^{\circ} \mathrm{Brix}$ ). El valor alcanzado por el tratamiento 2 sí fue significativamente superior con respecto a los tratamientos 3 y 4 (entre 10,90 y $11,15^{\circ}$ Brix). Por otra parte, no hubo diferencias significativas entre los tratamientos 1 , 3 y 4 entre sí, para esta característica.

Unos investigadores evaluaron el efecto de la aplicación vía foliar de tres fuentes de calcio en melón Honey Dew, y no hallaron diferencias entre tratamientos en cuatro localidades para el porcentaje de sólidos solubles totales, pero en otra localidad el tratamiento testigo sí obtuvo un mayor valor para esta variable en comparación con los tratamientos de aplicación de calcio [7]; en el presente ensayo se obtuvieron resultados diferentes a los hallados por dichos autores.

En otras investigaciones realizadas en Brasil y Argentina, no se presentaron diferencias en el porcentaje de sólidos solubles totales, entre el testigo y la aplicación de diferentes dosis y/o fuentes de calcio a nivel foliar, en melón Amarillo [8], [6], [5]; los datos hallados en el presente estudio fueron similares a los informados por esos autores, en el sentido de que el tratamiento testigo no fue estadísticamente diferente de los otros tratamientos evaluados. 




Figura 5. Porcentaje de sólidos solubles totales ( ${ }^{\circ}$ Brix), según tratamiento.

Nota: Medias seguidas de una misma letra no se consideran distintas según la prueba de LSD Fisher $(p \leq 0,05)$.

En la figura 6 se presentan los valores obtenidos para la firmeza de la pulpa del fruto. El tratamiento 4 produjo los frutos con la pulpa más firme $(28,38 \mathrm{~N})$, y el menor valor para esta característica correspondió al tratamiento 1 (testigo, con 26,94 N). Sin embargo, no hubo diferencias estadísticamente significativas entre tratamientos para esta variable.

En Argentina, se informó que la aplicación de tres fuentes de calcio vía foliar provocó el aumento de la firmeza de la pulpa del fruto en melón Honey Dew, en comparación con el testigo, en cuatro localidades; pero en otra localidad no hubo diferencias entre tratamientos [7], tal y como sucedió en el presente ensayo.

Unos investigadores hallaron diferencias en la firmeza del fruto de melón Amarillo entre el testigo y el tratamiento de aplicación foliar de nitrato de calcio a una dosis de 2,6 g/l; pero no se presentaron diferencias entre el testigo y las dosis de 1,3; 5,2; y 10,5 g/l de nitrato de calcio [8], de manera similar a los resultados hallados en el presente trabajo.

En otro ensayo con melón Amarillo en Brasil, la firmeza del fruto aumentó con la aplicación foliar de calcio, en comparación con el testigo [6].

Con respecto a la evaluación fitosanitaria, a los 12 ddt y a los 26 ddt, las plantas estaban completamente sanas y libres de enfermedades. A los $40 \mathrm{ddt}$, se encontró una incidencia importante de la enfermedad mildiú polvoso (Erysiphe sp. y/o Sphaerotheca sp.), distribuida uniformemente en todos los tratamientos. A los 50 ddt, el mildiú polvoso ya había sido controlado; además, se encontraron algunas plantas con síntomas de afectación por virus en las hojas, otras plantas mostraron un ataque leve de minador (larvas de la mosca Liriomyza sp.) en las hojas, y también se presentaron síntomas de la enfermedad mildiú velloso (Pseudoperonospora cubensis), pero que ya había sido controlada mediante fungicidas.

En la figura 7 se muestra la evaluación de la severidad máxima de mildiú velloso por tratamiento, a los 50 ddt. El tratamiento 2 fue el que mostró la menor severidad máxima de esta enfermedad, con una calificación de 3,83 puntos (escala 1-5), mientras que el mayor valor para esta variable correspondió a los tratamientos 3 y 4, con 4,17 puntos para cada uno. Sin embargo, no se presentaron diferencias estadísticamente significativas entre tratamientos para esta variable. En términos generales, estos resultados indican que las hojas mostraron una severidad máxima de 7-10 lesiones necróticas causadas por esta enfermedad a los 50 ddt, lo que se considera una severidad alta. 


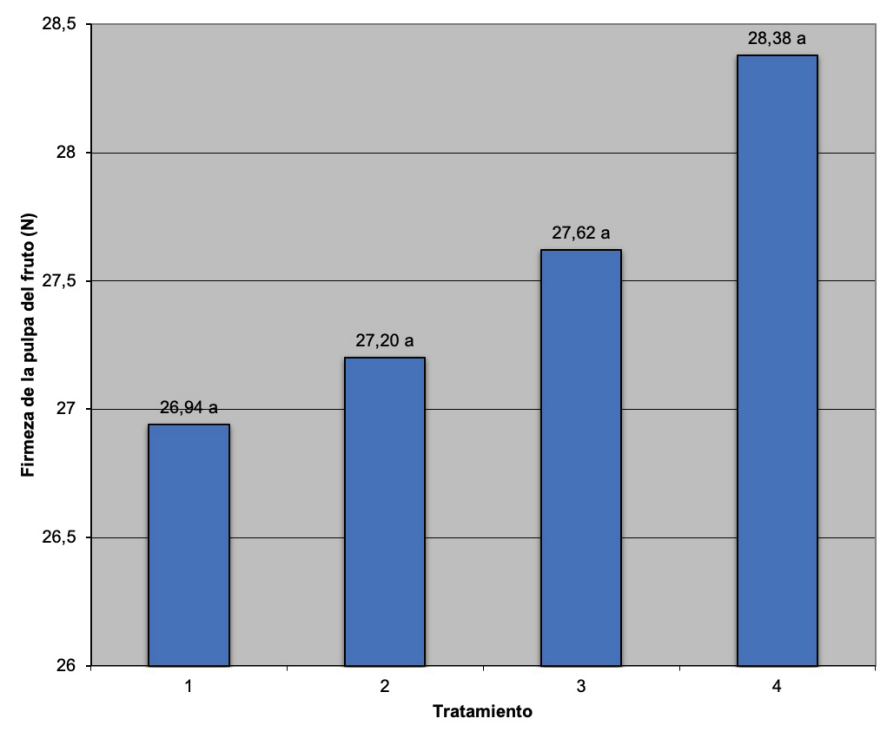

Figura 6. Firmeza de la pulpa del fruto (N), según tratamiento.

Nota: Medias seguidas de una misma letra no se consideran distintas según la prueba de LSD Fisher $(p \leq 0,05)$.

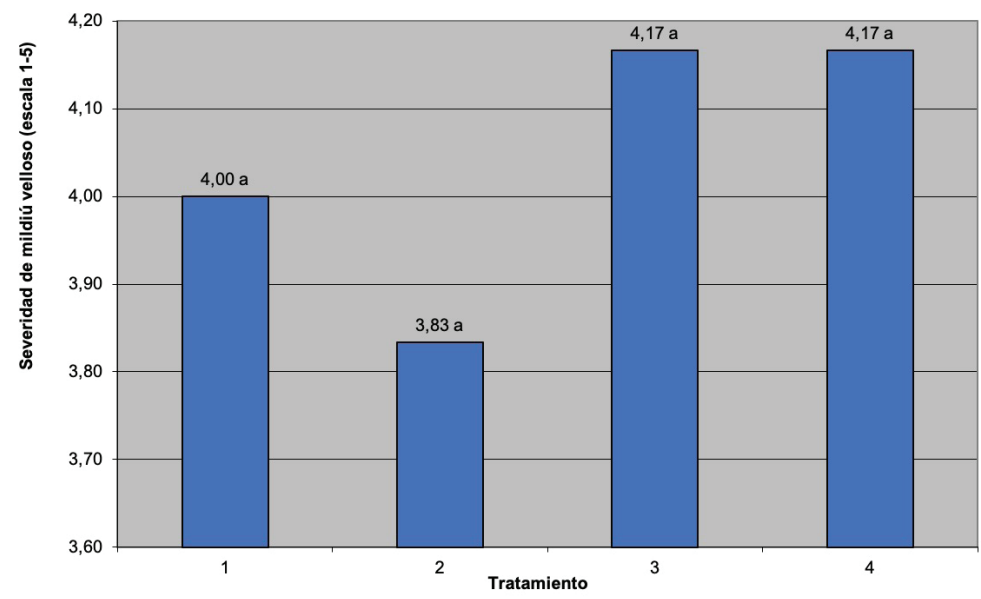

Figura 7. Severidad máxima de mildiú velloso (escala 1-5), a los 50 ddt, según tratamiento.

Nota: Medias seguidas de una misma letra no se consideran distintas según la prueba de LSD Fisher $(p \leq 0,05)$.

Al inicio de la cosecha (58 ddt), se encontraron algunas plantas colapsadas por enfermedades causadas por hongos fitopatógenos en el cuello de la planta; en la figura 8 se presentan los datos de la incidencia de esta enfermedad en ese momento. El tratamiento 2 fue el único que no mostró ninguna planta colapsada por dicha patología. Le siguió el tratamiento 3, que presentó una incidencia de 0,62 \%, el tratamiento 1 con 1,23\%, y el mayor valor para esta variable se obtuvo con el tratamiento 4 (1,85\%). Sin embargo, las diferencias halladas entre tratamientos no fueron estadísticamente significativas.

También, en el momento del inicio de la cosecha (58 ddt) se encontraron varios frutos que mostraban síntomas de afectación por virus fitopatógenos (como manchas y protuberancias), y otros frutos que presentaban daños en la cáscara causados por larvas de insectos (Spodoptera spp. y Diaphania spp.) Obviamente, esos frutos fueron descartados ya que no cumplían con los estándares para exportación. 


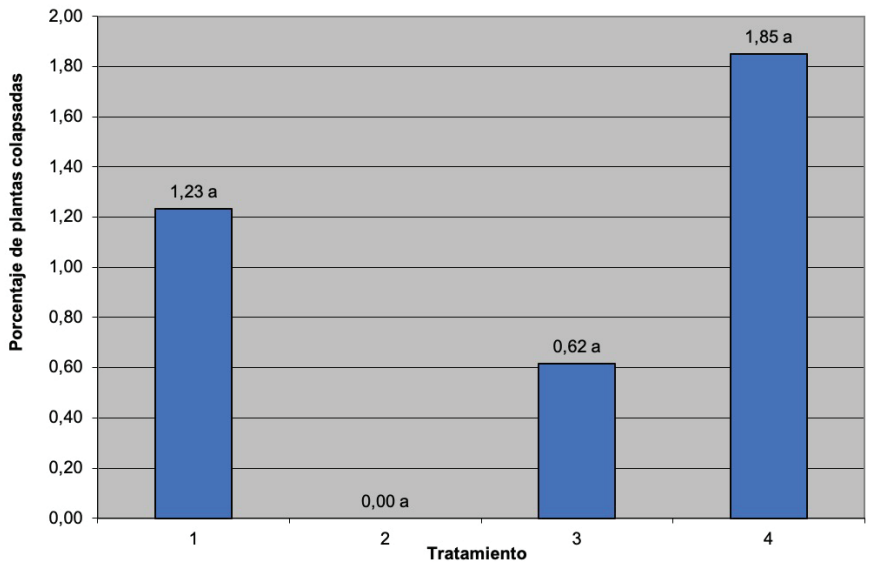

Figura 8. Incidencia de colapso de la planta (porcentaje), a los 58 ddt, según tratamiento.

Nota: Medias seguidas de una misma letra no se consideran distintas según la prueba de LSD Fisher $(p \leq 0,05)$.

En el cuadro 5 se presentan los resultados del análisis químico foliar para los cuatro tratamientos.

Según los rangos definidos por el Centro de Investigaciones Agronómicas de la Universidad de Costa Rica para el cultivo de melón [9], en todos los tratamientos se encontraron niveles adecuados (suficientes) en la mayoría de elementos (N, P, S, Fe, Zn, Mn y B). Solamente se presentaron niveles bajos en el caso del potasio (alrededor de la mitad de lo necesario), en los cuatro tratamientos. Por otra parte, se presentaron niveles altos en el caso del cobre, magnesio y calcio en todos los tratamientos; particularmente en el caso del calcio, el nivel obtenido casi llega a duplicar el nivel considerado suficiente, lo que podría explicar por qué no hubo respuesta a los tratamientos de aplicación foliar suplementaria de calcio evaluados en este ensayo, para la mayoría de las variables evaluadas.

Sin embargo, otra investigadora considera como normal en melón que el contenido foliar de $\mathrm{N}$ y $\mathrm{K}$ sea de alrededor del $3 \%$ para cada uno de ellos, y que el contenido foliar de Ca sea de alrededor del $5 \%$ [3]. Bajo estos parámetros, los resultados encontrados en el presente ensayo se ajustan mejor a los valores considerados normales, excepto que se presentó un exceso de $\mathrm{N}$ con respecto a $\mathrm{K}$, lo que podría haber afectado la producción de frutos, y haber favorecido más bien el crecimiento vegetativo.

Definitivamente, el bajo rendimiento exportable hallado en el presente ensayo podría estar asociado al bajo nivel de potasio encontrado en las hojas del melón. El potasio es un elemento esencial para lograr el llenado del fruto [10]. Este bajo nivel de potasio encontrado a nivel foliar podría ser el factor limitante más importante para el cultivo en este caso. Lo normal es que, en el tejido vegetativo de melón, la concentración de $\mathrm{K}$ sea mayor que la de $\mathrm{N}$ [3], pero en el presente ensayo se encontró la situación contraria. Sin embargo, el bajo rendimiento obtenido en este caso también podría estar relacionado con otros factores limitantes ya comentados, como el efecto de plagas y enfermedades, en especial con la enfermedad mildiú velloso, que presentó una severidad máxima alta a los 50 ddt. 
Cuadro 5. Resultados del análisis químico foliar, según tratamiento.

\begin{tabular}{|c|c|c|c|c|c|c|c|}
\hline \multirow{2}{*}{ Elemento } & \multirow{2}{*}{ Unidad } & \multicolumn{4}{|c|}{ Tratamiento } & \multirow{2}{*}{ Rango suficiente* } & \multirow{2}{*}{ Diagnóstico } \\
\hline & & 1 & 2 & 3 & 4 & & \\
\hline$N$ & $\%$ & 4,79 & 5,02 & 4,81 & 4,83 & $4,5-5,5$ & suficiente \\
\hline$P$ & $\%$ & 0,52 & 0,52 & 0,51 & 0,48 & $0,3-0,8$ & suficiente \\
\hline $\mathrm{Ca}$ & $\%$ & 4,28 & 4,23 & 4,81 & 4,98 & $2,3-3,0$ & alto \\
\hline $\mathrm{Mg}$ & $\%$ & 0,91 & 0,91 & 1,02 & 1,02 & $0,35-0,8$ & alto \\
\hline K & $\%$ & 2,32 & 2,17 & 2,41 & 2,43 & $4,0-5,0$ & bajo \\
\hline$S$ & $\%$ & 0,78 & 0,82 & 0,82 & 0,74 & $0,25-1,0$ & suficiente \\
\hline $\mathrm{Fe}$ & $\mathrm{mg} / \mathrm{kg}$ & 175 & 235 & 256 & 265 & $50-300$ & suficiente \\
\hline $\mathrm{Cu}$ & $\mathrm{mg} / \mathrm{kg}$ & 35 & 38 & 34 & 36 & $8,0-30$ & alto \\
\hline $\mathrm{Zn}$ & $\mathrm{mg} / \mathrm{kg}$ & 47 & 49 & 47 & 46 & $20-200$ & suficiente \\
\hline $\mathrm{Mn}$ & $\mathrm{mg} / \mathrm{kg}$ & 101 & 106 & 103 & 102 & $50-250$ & suficiente \\
\hline B & $\mathrm{mg} / \mathrm{kg}$ & 38 & 39 & 40 & 41 & $25-60$ & suficiente \\
\hline
\end{tabular}

*Fuente: [9].

En el cuadro 6 se presenta la relación beneficio:costo para los tratamientos de aplicación foliar suplementaria de calcio, en comparación con el tratamiento testigo.

Cuadro 6. Relación beneficio:costo, según tratamiento.

\begin{tabular}{|c|c|c|c|}
\hline Tratamiento & Beneficios adicionales (colones/ha) & Costos adicionales (colones/ha) & Relación beneficio:costo \\
\hline 1 & n.a. & n.a. & n.a. \\
\hline 2 & 315.744 & 76.000 & $4,15: 1$ \\
\hline 3 & -647.680 & 43.429 & $-14,91: 1$ \\
\hline 4 & -153.824 & 50.127 & $-3,07: 1$ \\
\hline
\end{tabular}

El único caso en que el beneficio adicional estimado fue superior al costo correspondió al tratamiento 2 (relación beneficio:costo de 4,15), en el cual el retorno de la inversión fue de 4,15 colones por cada colón invertido, lo que se considera una opción altamente rentable. Los tratamientos 3 y 4 obtuvieron una relación beneficio:costo negativa, por lo que no se consideran económicamente rentables. Sin embargo, se debe recordar que no se presentaron diferencias estadísticamente significativas en la producción exportable en cajas por hectárea, entre tratamientos.

\section{Conclusiones y recomendaciones}

Se presentaron diferencias estadísticamente significativas entre tratamientos para el porcentaje de sólidos solubles totales. El tratamiento 2 (JMX-Ca a 4,22 kg CaO/ha) produjo frutos con un valor significativamente más alto (11,88 ${ }^{\circ}$ Brix) para esta variable, en comparación con los tratamientos 3 (JMX-Ca a 2,41 kg CaO/ha) y 4 (Manvert-Ca a 4,22 kg CaO/ha) (entre 10,90 y 
$11,15^{\circ}$ Brix). Sin embargo, el resultado obtenido por el tratamiento 2 no fue estadísticamente diferente del valor hallado por el tratamiento 1 (testigo, 11,45 ${ }^{\circ}$ Brix).

A pesar de que en todas las demás variables evaluadas no se encontraron diferencias estadísticamente significativas entre tratamientos, prácticamente en todos los casos el mejor resultado fue obtenido por el tratamiento 2 (JMX-Ca a 4,22 kg CaO/ha), excepto en la firmeza del fruto, en que el mejor resultado se obtuvo con el tratamiento 4 (Manvert-Ca a 4,22 kg CaO/ha).

Se concluye que, bajo las condiciones en que se desarrolló el ensayo, no se justifica económicamente la aplicación foliar suplementaria de calcio.

\section{Agradecimientos}

Los autores agradecen el apoyo de la empresa M y M Internacional S. A. y de su personal, para la ejecución del trabajo. Asimismo, agradecen la colaboración de Gabriela Zárate, Jendry Portilla y Jorge Díaz en el trabajo de campo.

\section{Referencias}

[1] J. E. Monge-Pérez, «Producción y exportación de melón (Cucumis melo) en Costa Rica,» Tecnología en Marcha, vol. 27, n 1, pp. 93-103, 2014.

[2] J. E. Monge-Pérez, «Caracterización económica de la producción de melón (Cucumis melo) en Costa Rica,» de La investigación en Guanacaste II, San José, Costa Rica, Nuevas Perspectivas, 2016, pp. 257-275.

[3] F. Bertsch, «Utilización de estudios de crecimiento y absorción de nutrimentos para afinar programas de fertilización foliar,» de Fertilización foliar: principios y aplicaciones, San Pedro de Montes de Oca, San José, Costa Rica, CIA-UCR-ACCS, 2002, pp. 129-146.

[4] L. Rincón, «Fertilización del melón en riego por goteo,» de Melones, Vols. \%1 de \%2Compendios de Horticultura, 10, Reus, España, Ediciones de Horticultura S. L., 1997, pp. 85-93.

[5] J. E. Yuri, T. S. D. Ferreira, D. R. S. Gama, G. M. d. Resende y J. H. Mota, «Produtividade e qualidade dos frutos de melão pulverizados com cálcio,» Horticultura Brasileira, vol. 30, n 2 (Suplemento-CD Rom), pp. S6563-S6569, 2012.

[6] A. J. Pereira, A. F. Blank, M. A. R. Alvarenga y R. J. d. Souza, «Aplicação de fontes e doses de cálcio na produção e qualidade de frutos de melão,» Horticultura Brasileira, vol. 20, nº 3, pp. 428-431, 2002.

[7] C. A. Bouzo y S. B. Cortez, «Efecto de la aplicación foliar de calcio sobre algunos atributos de calidad en frutos de melón,» RIA, vol. 38, n³, pp. 257-262, 2012.

[8] F. F. Muñoz, V. E. Ruiz y C. A. Bouzo, «Effect of preharvest foliar sprays of calcium nitrate on melon fruit quality,» Pyton, vol. 86, pp. 131-136, 2017.

[9] Laboratorio de Suelos y Foliares, «Tabla de interpretación de análisis foliar en melón,» Centro de Investigaciones Agronómicas, Universidad de Costa Rica, San José, Costa Rica, 2002.

[10] F. Bertsch, La fertilidad de los suelos y su manejo, San José, Costa Rica: ACCS, 1995, p. 157. 